\title{
Molecules that swim, or not
}

\author{
The reported observation of enhanced diffusivity of simple molecules during a chemical reaction in solution is but \\ the latest of nanoscale wonders.
}

( an a chemical reaction propel a molecule in solution? Or, in technical jargon, does (or even, can) the diffusivity of a molecule change during a reaction? The jury is still out, but evidence is mounting for this surprising phenomenon to be real.

Diffusivity, or the diffusion coefficient, measures the amount of molecules passing through a surface per unit of time. It is measured in units of $\mathrm{m}^{2} \mathrm{~s}^{-1}$. The diffusion coefficient depends on intrinsic properties of the molecule (diameter) and its surroundings (viscosity of the solution, temperature) through the ubiquitous Stokes-Einstein equation.

Now, in a series of experiments, Steve Granick and colleagues from the Institute for Basic Science in Ulsan (South Korea) and Granick's former postdoc, Huan Wang, presently at Peking University (China), observed that molecules diffuse faster (or, swim!) as they react. Depending on the reaction, the change of diffusivity was in the order of 1 to $10 \%$, with the larger effect observed in catalysed bimolecular reactions (for example, an alkyne-azide click reaction) and the smaller end for nucleophilic substitution reactions $\left(\mathrm{S}_{\mathrm{N}} 1\right.$ and $\left.\mathrm{S}_{\mathrm{N}} 2\right)$ (refs. $\left.{ }^{1,2}\right)$.

In a particularly elegant experiment, the team used a microfluidic setup. The chamber contained a constant concentration of a dye molecule, acting as a tracer; and a concentration gradient of a catalyst along the length of the reactor. As the reactant was added, uniformly along the chamber, a concentration gradient of the tracer began to develop, but with opposite slope with respect to the concentration gradient of the catalyst. The effect stopped as the reaction ended. The authors posited that a backflow of solvent (and dye) molecules must occur as a result of an enhanced diffusivity of the molecules involved in the reaction in order to keep the fluid density constant in the microfluidic chamber. In other words, as more molecules move towards the regions of high catalyst concentration, solvent molecules will occupy the space left behind by the reactant.

In the past couple of decades, a whole research field has developed that deals with microswimmers in which particles' motion in solution can be directed by a whole set of external parameters ${ }^{3}$. Particles showing phototaxis, chemotaxis, or propelled in

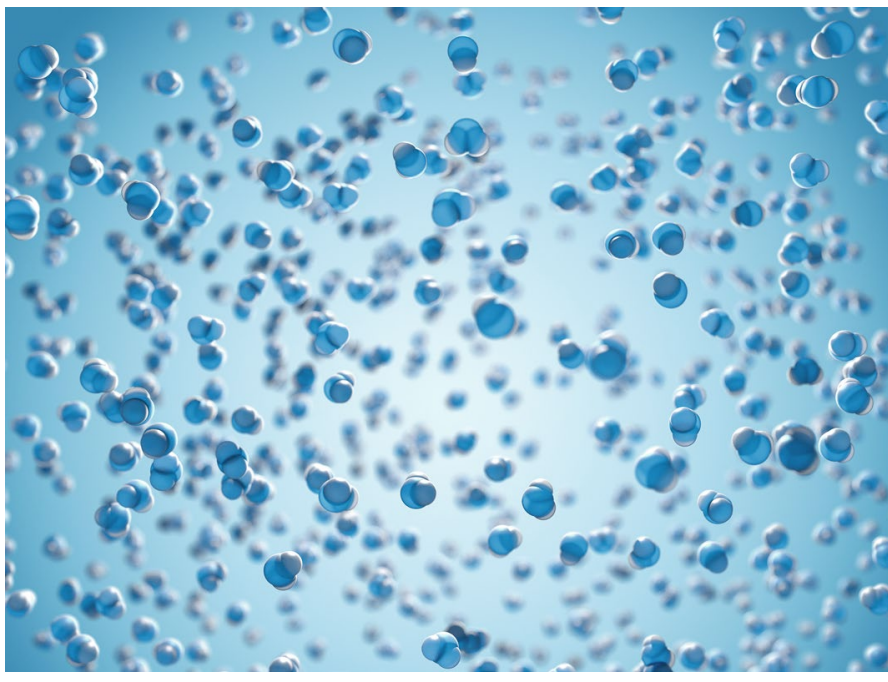

Credit: Science Lab / Alamy Stock Photo

solution by the formation of bubbles, have been demonstrated. One lofty goal is to engineer drug delivery systems that can sense a specific gradient in the body and follow it to release a cargo at the target. Nanoparticle diffusion can also be controlled, although this is already much harder than for microparticles because, due to their smaller size, Brownian fluctuations start to kick in a big way, randomizing directed propulsion. Here, like in all the other nanoscale phenomena, size counts.

Therefore, pushing oneself to hypothesize that objects as small as a molecule could show enhanced diffusion is a huge conceptual leap. Although a pioneering study had stumbled onto something related $^{4}$, Granick decided to investigate the phenomenon systematically. After some convincing, students and postdocs, including Wang, decided to give it a go. What followed was a period of disbelief, heated discussions, repeated experiments, countless controls, and error-bar analyses; but when the diffusivity enhancement was reproducible in a whole set of chemical reactions, it was time to publish ${ }^{1,2}$.

Based on these results, saying that molecules in solution during a chemical reaction strictly obey the Stokes-Einstein equation may be physically wrong, or at least not the full story; but, so far, this assumption has held fairly well for many intents and purposes. "You don't notice a new phenomenon, until you do the right measurements." says Granick. Still, he admits that the mechanism remains unclear: efforts to correlate diffusivity enhancement with the reaction rate or the size of the transition state complex are still inconclusive. As with any strange and unexpected effect with a mechanism that is not immediately obvious, the observations of Granick and Wang have also raised criticism ${ }^{5}$.

However, whether or not reacting molecules are the ultimate nanomotors, this debate is a contemporary, fascinating and intellectual frontier of bioinspired nanoscience research. At the end of it, we will develop new terminology to describe these systems that currently defy accepted models. Molecular motors in natural systems efficiently couple chemical energy to kinetic energy and direct transport of matter in cells, despite the randomness of Brownian motion. Hence, there is no physical reason to doubt that we will also be able to understand and harness randomness in synthetic nanosystems, to our benefit.

Published online: 10 November 2021 https://doi.org/10.1038/s41565-021-01035-7

References

1. Wang, H. et al. Science 369, 537-541 (2020).

2. Huang, T. et al. ACS Nano 15, 14947-14953 (2021).

3. Zhang, Y. \& Hess, H. Nat. Rev. Chem. 5, 500-510 (2021).

4. Pavlick, R. A. et al. Nanoscale 5, 1301-1304 (2013).

5. Günther, J.-P. et al. Science 371, eabe8322 (2021). 